\title{
Drop Metastasis Seeding the Intramedullary Conus Medullaris in a Patient with Breast Cancer and Brain Metastasis
}

\author{
Jung-Ying Chiang ${ }^{1}$ and Der-cherng Chen ${ }^{1,2 *}$ \\ ${ }^{1}$ Department of Neurosurgery, China Medical University Hospital, Taichung, Taiwan, R.O.C \\ ${ }^{2}$ China Medical University, Taichung, Taiwan, R.O.C
}

\begin{abstract}
Breast cancer metastasis to the conus medullaris is very rare. We report a case of intra-axial conus medullaris metastasis from breast adenocarcinoma in a 39-year-old woman. Two years after the diagnosis of brain metastasis was biopsy-proven, she developed progressive lower limb weakness with urinary incontinence for one month. MRI revealed a contrast-enhancing intramedullary lesion at the conus medullaris. Laminectomy and removal of the tumor were performed for decompression and for preserving function. Postoperative histopathological examinations confirmed the metastatic nature of the lesion. We discuss the possibility of drop metastasis traveling from the brain to the conus medullaris via cerebrospinal fluid (CSF) in the central canal. Since intramedullary spinal cord metastasis can lead to rapid progressive neurological deficits and poor outcome, early diagnosis and decompression could preserve the quality of life. We suggest that CSF cytology could be helpful for the diagnosis of intraspinal metastasis in patients with brain metastasis.
\end{abstract}

Keywords: Intramedullary; Spinal cord; Metastasis; Breast cancer

\section{Introduction}

Intramedullary spinal cord metastasis (ISCM) is rare (representing approximately $0.1-0.4 \%$ of all spinal cord neoplasms [1]), and nearly $50 \%$ of ISCMs arise from primary lung cancer. Other primary sources include primary breast and colorectal cancers [2]. Spinal magnetic resonance imaging (MRI) is routinely used to diagnose ISCM [3]. Surgical excision aims at decompression of functional neural tissue and at histological confirmation of the diagnosis [4]. We present a case of intramedullary conus medullaris metastasis from primary breast adenocarcinoma.

\section{Case Report}

A 39-year-old premenopausal woman presented with T2N0M0 left breast cancer treated in 1997 with surgery followed by anthracyclinebased chemotherapy and tamoxifen. The tumor was Her-2/neu (3+) and estrogen/progesterone receptor negative. She achieved a complete response and was then followed up on a regular basis. Metastasis to the lung developed in 2009 and then progressed to the liver and brain, and was treated with herceptin, avastin, and cisplatin. Brain biopsy was performed to pathologically confirm the diagnosis of brain metastasis, which was treated with whole brain radiation. However, weakness, paresthesia, and numbness in the lower extremities had progressed two years after the brain biopsy leaving the patient unable to walk and urinary incontinent. The MRI of the lumbar spine disclosed a 3.9 $\times 1.4 \mathrm{~cm}$ mass within the medullary conus at spinal level T12 to L1. T1- and T2-weighted sequences showed significant enhancement of the heterogeneous hyperintense lesion after gadolinium injection, no bony destruction, and no epidural tumors (Figure 1). Muscle strength decreased from 5 to 2 , and urinary incontinence persisted. Laminectomy of T12 and L1 and durotomy were performed, and a medullary conus tumor was found. During partial resection of the medullary conus tumor, the lumen of the central canal was seen to be patent and to have a smooth surface. The nerve roots of the cauda equina were released using microsurgical techniques. The patient had no notable complications; her lower limb weakness and numbness improved immediately after the operation, but she was still unable to walk and remained dependent on a Foley catheter. Postoperatively, she underwent spinal local radiotherapy ( $30 \mathrm{~Gy} / 12 \mathrm{Fr})$ and further rehabilitation.
Histological and genetic findings: Microscopic examination showed metastatic adenocarcinoma composed of polygonal tumor cells with pleomorphic and hyperchromatic nuclei arranged in vague glandular structures or nests (Figure 2). Immunohistochemical study showed an immunophenotype consistent with breast cancer, i.e., CK7 $(+)$, CK20 (-), TTF-1(+), ER (-), PR (-), and Her-2/Neu (weakly positive, score $2+$ ).

\section{Discussion}

The treatment of ISCM could be steroids, radiation, chemotherapy,

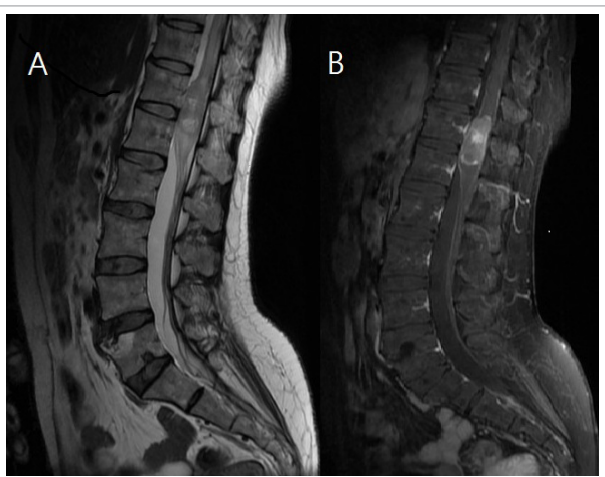

Figure 1: The sagittal T2-weighted spinal MRI and sagittal T1-weighted spinal MRI (A) with contrast (B) showing an intra-axial mass at the level of the conus medullaris, measuring approximately $3.9 \times 1.4 \mathrm{~cm}$. The cord focally expands at this level with edema extending both inferiorly and superiorly.

*Corresponding author: Der-cherng Chen, Assistant Professor of Medicine, Department of Medicine, China Medical University; Department of Neurosurgery, China Medical University Hospital, No. 2 Yu-Der Road, Taichung 404, Taiwan, R.O.C, Tel: +886-42-20521215034; Fax: +886-42-2052121x5035; E-mail: vincenchen1966@gmail.com

Received July 12, 2013; Accepted July 31, 2013; Published August 05, 2013

Citation: Chiang JY, Chen DC (2013) Drop Metastasis Seeding the Intramedullary Conus Medullaris in a Patient with Breast Cancer and Brain Metastasis. J Gen Pract 1: 119. doi: 10.4172/2329-9126.1000119

Copyright: (c) 2013 Chiang JY, et al. This is an open-access article distributed under the terms of the Creative Commons Attribution License, which permits unrestricted use, distribution, and reproduction in any medium, provided the original author and source are credited. 


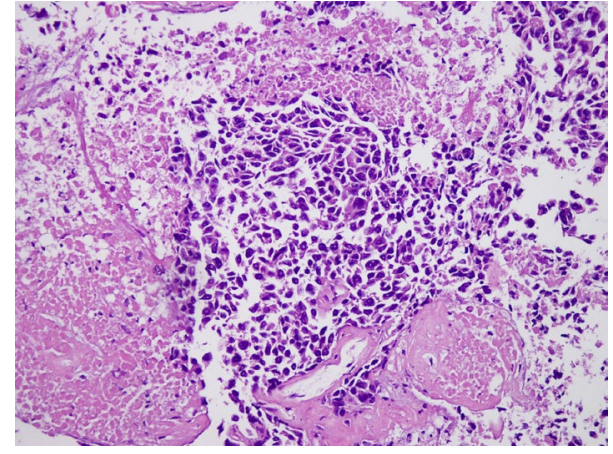

Figure 2: Microscopic view shows metastatic adenocarcinoma composed of polygonal tumor cells with pleomorphic and hyperchromatic nuclei arranged in a vague glandular structure or nests (magnification 200x).

and surgery. It remains controversial [5]. ISCM can be surgically excised since most ISCMs are well encapsulated. Although the surgery does not improve survival duration, radical removal of the intramedullary metastasis to the conus medullaris may improve quality of life [6,7]. The risks of operative morbidity remain high as this site is a transition point from the spinal cord to nerve roots, and a circumscribed mass may not be sharply demarcated. Partial resection may be advocated with the goal of preserving existing function when complete resection is impossible [8]. In the present case, the mass lesion was seen at the opening of the central canal and found to be well encapsulated. After removal of the tumor, the CSF flow and patency of the central canal were noted. To our knowledge, this is the first intramedullary spinal cord conus medullaris metastasis from a breast adenocarcinoma to be found in a patient with brain metastasis. We suspected that the ISCM was due to a drop metastasis from the brain metastasis that seeded the intramedullary space of the spinal cord. ISCMs are mainly disseminated by the arterial route. Meningeal carcinomatosis occurs by CSF seeding. Tumor cells infiltrate the Virchow-Robin spaces of vessels, penetrating the spinal cord and pial membrane and invading the spinal cord parenchyma. A third mechanism is the direct invasion from contiguous structures. Spread through the vertebral venous plexus (Batson's plexus) during the Valsalva manoeuvre enabling retrograde blood flow to the spinal cord is also possible [2]. But the three mechanisms about the pathophysiology of metastasis to spinal cord are no clear. According to the literature, ISCMs co-occur with meningeal carcinomatosis in $15-55 \%$ of cases [9]. At the time of the diagnosis, systemic metastases are present in most ISCM cases and brain metastases are present in nearly half of ISCM cases [3].

Rapid progression of symptoms distinguishes ISCM from primary intramedullary tumors, which typically present with a slower progression of symptoms [10]. The CSF seeding pathway has been mentioned in one study of meningioma with metastasis that used CSF sampling to detect meningioma metastasis [11] and two studies of drop metastases from anaplastic oligoendroglioma [12,13]. As in the above cases, CSF cytology should be helpful in cases like the one described herein for differential diagnosis preoperatively. And CSF cytology could support the diagnosis without requiring tissue excision for pathology, especially in those patients who refuse surgery in the terminal stages of their disease. Moreover, they could receive radiation therapy and chemotherapy to control the primary cancer. Nevertheless, in those cases of rapidly progressive neurological deficits, surgery could be considered as primary treatment whenever feasible. So we suggest CSF cytology could be routinely performed when intramedullary spinal metastasis is suspected. CSF cytology could also be helpful for detection of metastasis before symptoms become apparent in those patients with prior brain metastasis. But the value of CSF sampling needs to be confirmed in more studies.

\section{Conclusion}

To our knowledge, we report here the first case of successful surgical treatment of intramedullary spinal cord metastasis to the conus medullaris from a breast adenocarcinoma in a patient with brain metastasis. Treatment could be surgery, conservative, palliative measures, corticosteroids, chemotherapy, or radiotherapy. CSF cytology could help make the diagnosis without the need to obtain a surgical specimen for pathology and may detect the intramedullary spinal metastasis before the symptoms become apparent in those patients with prior brain metastasis.

\section{References}

1. Hsu KC, Li TY, Chu HY, Chen LC, Chang ST, et al. (2012) Conus medullaris metastasis in breast cancer: report of a case and a review of the literature. Surg Today 43: 910-914

2. Kalayci M, Cağavi F, Gül S, Yenidünya S, Açikgöz B (2004) Intramedullary spinal cord metastases: diagnosis and treatment-an illustrated review. Acta Neurochirurgica 146: 1347-1354

3. Schiff D, O'Neill BP (1996) Intramedullary spinal cord metastases: clinica features and treatment outcome. Neurology 47: 906-912.

4. Gasser T, Sandalcioglu IE, El Hamalawi B, van de Nes JA, Stolke D et al. (2005) Surgical treatment of intramedullary spinal cord metastases of systemic cancer: functional outcome and prognosis. J Neuro-Oncology 73: 163-168.

5. Guppy KH, Wagner $\mathrm{F}$ (2006) Metastasis to the conus medullaris: case report Neurosurgery 59: E1148.

6. Hrabalek L, Kalita O, Studentova H, Jankova J, Ehrmann J, et al. (2010) Intramedullary spinal cord and cauda equina metastasis of breast carcinoma: case report. Biomed Pap Med Fac Univ Palacky Olomouc Czech Repub 154 $175-177$.

7. Rostami R, Sfarpour D, Tavassoli FA, Jabbari B (2013) Intramedullary metastasis in breast cancer-A comprehensive literature review. J Neurol Sci

8. Dam-Hieu P, Seizeur R, Mineo J-F, Metges JP, Meriot P, et al. (2009) Retrospective study of 19 patients with intramedullary spinal cord metastasis. Clin Neurol Neurosurg 111: 10-17.

9. Kalita O (2011) Current insights into surgery for intramedullary spinal cord metastases: a literature review. Int J Surg Oncol.

10. Ogino M, Ueda R, Nakatsukasa M, Murase I (2002) Successful removal of solitary intramedullary spinal cord metastasis from colon cancer. Clin Neurol Neurosurg 104: 152-156

11. Erkutlu I, Buyukhatipoglu H, Alptekin M, Berkyurek E, Tutar E (2009) Spinal drop metastases from a papillary meningioma: a case report and review of the literature: utility of CSF sampling. Med Oncol 26: 242-246.

12. Carlsen JG, Tietze A, Lassen YA, Rosendal F (2012) Paraplegia due to drop metastases from anaplastic oligodendroglioma. Brit J Neurosurg 26: 94-95.

13. Natale M, Spennato P, Savarese L, Bocchetti A, Esposito S et al. (2005) Anaplastic oligodendroglioma presenting with drop metastases in the cauda equina. Clin Neurol Neurosurg 107: 417-420. 